\title{
Research Review of Fault Diagnosis Method for High Engine Water Temperature
}

\author{
Feng Xia
}

Wuxi Vocational Institute of Commerce, Wuxi, Jiangsu 214153, China

Key words: High water temperature; Fault diagnosis; Fan module

\begin{abstract}
There are many reasons for the high water temperature of the engine, such as cooling fan not working, fan control module circuit failure, insufficient cooling medium and PCM failure. Primarily based on the analysis of the engine failure in this paper, the reasons for the failures were found out, and the fault diagnosis and maintenance method for high water temperature of the engine was checked with the adoption of real vehicle testing.
\end{abstract}

\section{Introduction}

When the engine overheats, it will decrease the power performance of the engine, and even damage the other parts of the engine if serious. When the water temperature is prompted to be high on the dashboard, the reason for the high temperature of the engine shall be first and foremost determined. According to the fault analysis result, the failure cause shall be gradually determined and the faulty part is found out.

\section{Failure Analysis}

There are many reasons for the high water temperature, such as fan belt fracture, water tank or water pipe leakage, and thermostat failure. If the engine is running out of power or spinning out of a violent knock or cylinder knocking, the engine may be overheating. The specific reasons are as follows:

Cooling Fan Failure. The cooling fan is controlled by a temperature control switch or controlled by water temperature sensor[1].The cooling fan failure is usually manifested as being unable to rotate, not rotating at a low speed and not rotating at high speed, and the circuit shall be analyzed for this kind of failure step by step to determine the failure.

Insufficient Cooling Medium. After a vehicle has run for a long time, the problem of insufficient cooling fluid will take place, and as for normal wear and tear, attention shall be just paid to adding the cooling medium in a timely manner; if there are problems such as the tank cover leakage, the corresponding parts will be replaced in a timely manner.

Damage to the Thermostat. The damage of the thermostat can not timely change the circulation route of cooling water[2]. If the thermostat fails, it should be replaced in time.

The Water Tank is Blocked. Where the outside of water the tank is blocked, the dirt shall be removed by use of water or gas; In case of the internal blockage of the water tank, it can affect the circulation of the coolant, while this will cause pressure in the water pipe[3], which will affect the circulation of the cooling water.

Water Pump Failure. When the pump fails[4], the impeller will slip or get stuck, so that the belt will be broken or the impeller will be deformed. If the water pump fails, it should be replaced immediately.

Cylinder Ablation. When the cylinder is ablated, the following phenomena may occur: (1) The combustion chamber is connected with water channel, when water or white smoke will be discharged from the drainage pipe, resulting in constant water shortage of the water tank [5]. (2)The water channel is connected with oil channel, resulting in white water in the oil. (3)The water channel communicates with water channel, resulting in poor heat dissipation of the cylinder body, which is more influential especially at a high speed. (4)The water channel is blocked. 
Other electrical control system failures, such as tire pressure monitoring and computer failure[6].

\section{Real Vehicle Testing}

The 2013 Ford Focus C346, 1.6MT has a high water temperature, and the mileage was 33650KM. The water temperature was high, so it was returned to the plant. According to the troubleshooting, the maintenance process is as follows:

(1)After the vehicle was returned to the factory because of the high water temperature, the electronic fan was found not to turn, and the fan did not run when turning on the air conditioning fan [7]. No fault code was checked by connecting IDS; when the water temperature reached 99 degrees through the data stream display, the PCM issued a fan operation instruction, but the fan did not turn, and by mechanic analysis, it was nothing but the fan itself faulty, line failure [8], or PCM failure.

(2)Check the wiring diagram as shown in figure 1. Check and confirm that the power supply of the fan module was normal, and it was also connected from No.4 pin of C1E203 plug to No.15 pin of C90 plug [9]. Therefore, the fan might have a problem. After a new fan was borrowed from the warehouse and was plugged, the fan did not turn by opening the air conditioning fan. It was also normal that the line of the fan module to PCM was re-checked.

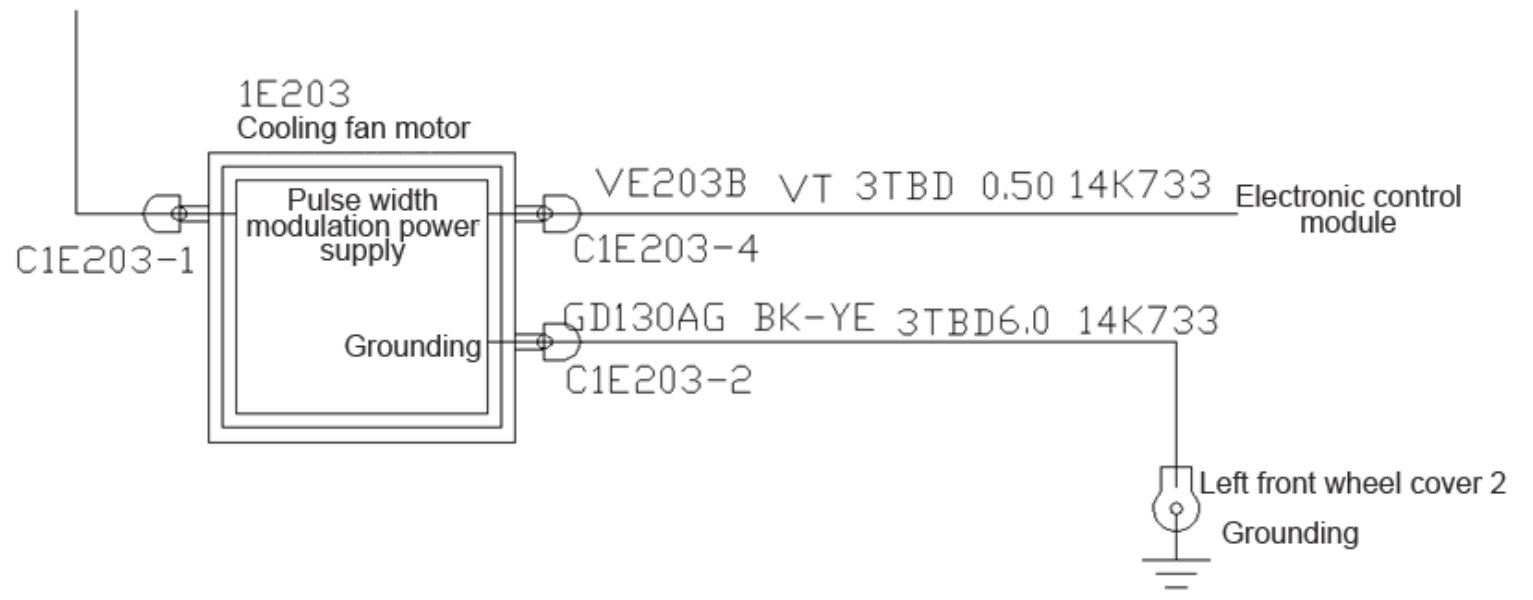

FIG. 1 Fan control module circuit

(3)Since there was no problem with the line by inspection, PCM failure was suspected; then, a normal PCM was changed, and the fan did not turn in the end [10]. Among the parts that caused the failure, all the others were replaced except the wiring harness, but the problem was not solved yet.

(4)At this time, I would like to check the waveform of Pin 15 of the C90 plug with the oscilloscope function of VMM, to confirm the faulty point [11]. The waveform detected by the faulty car is shown in figure 2. By using a normal car to detect the waveform, the waveforms were shown in FIG. 3, 4 and 5 when the fan does not run, starts operation and is in high-speed operation.

(5)That the fan does not turn is because no pulse instruction needed for operation was received, but the PCM has issued instructions; by checking that the circuit from the PCM to the fan module is conducted, the line inspection was focused on [12], that is, it was found that No.15 terminal of C90 plug was in poor contact. The reason for the fault then seemed to be found, but the terminal was processed, and the desired waveform was not seen after confirming good contact. However, the fault remained unresolved. In order to remove the poor contact of PCM pin, it was still necessary to dismantle the engine wiring harness of a normal car to verify whether the fault was as ever.

(6)The accessories in trouble have been changed, but the problem has not been solved; when rearranging the ideas, the car tire pressure monitoring was noticed, but it was not known whether monitoring the tire pressure would not affect the fan not turning [13], or the BCM of a normal car was attempted to be changed to the faulty car, it would start by opening the air conditioning fan immediately after starting the programming operation. In order to confirm that the BCM was faulty, the BCM of the faulty car was installed onto a normal car again, and it was confirmed that the fan doesn't work, after the BCM failure was identified, the customer was notified that because the 
program upgrading resulted in the damage to BCM, it needed to be replaced at the customer's own expense, and an order has been placed after consent by the customer. A new BCM was installed onto the faulty car to move the key and start the module, before thorough troubleshooting.

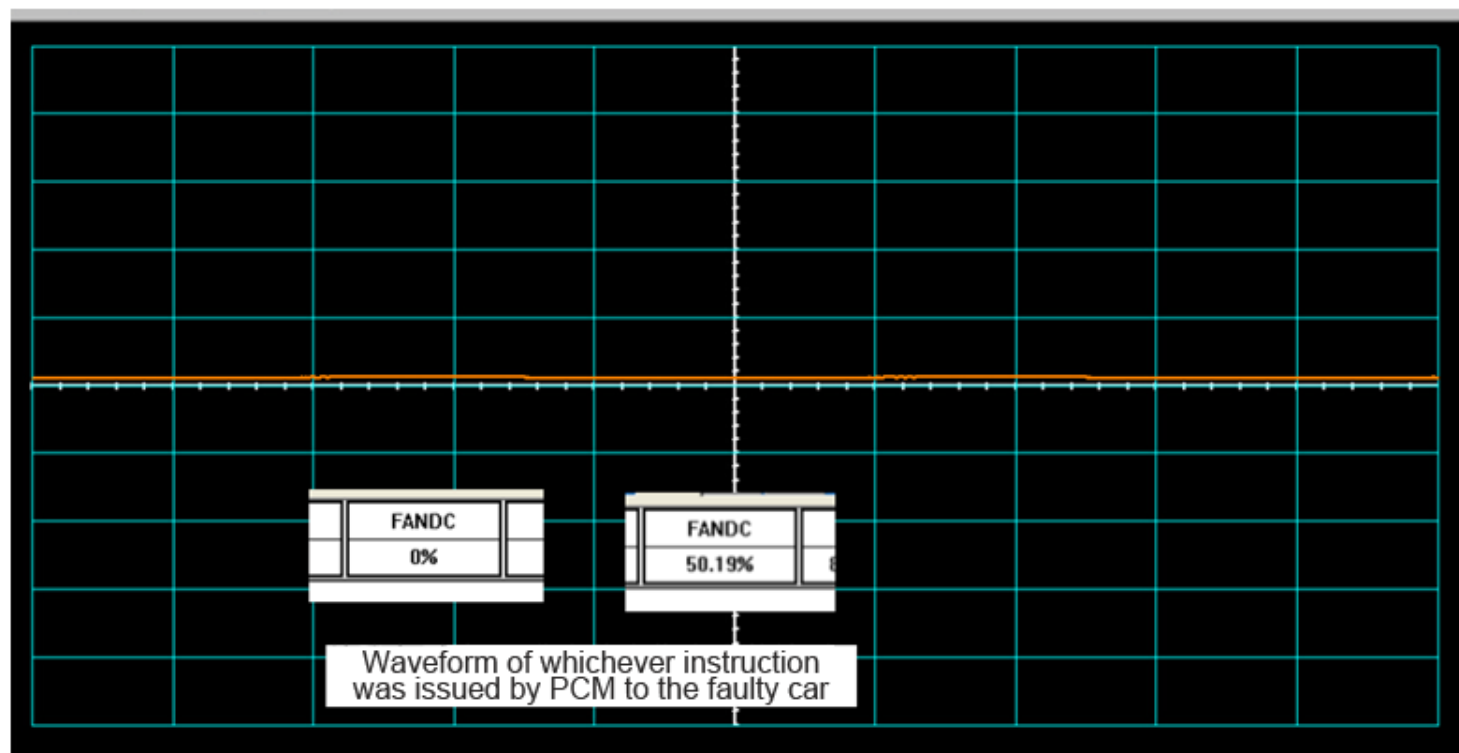

FIG. 2 Waveform of faulty car

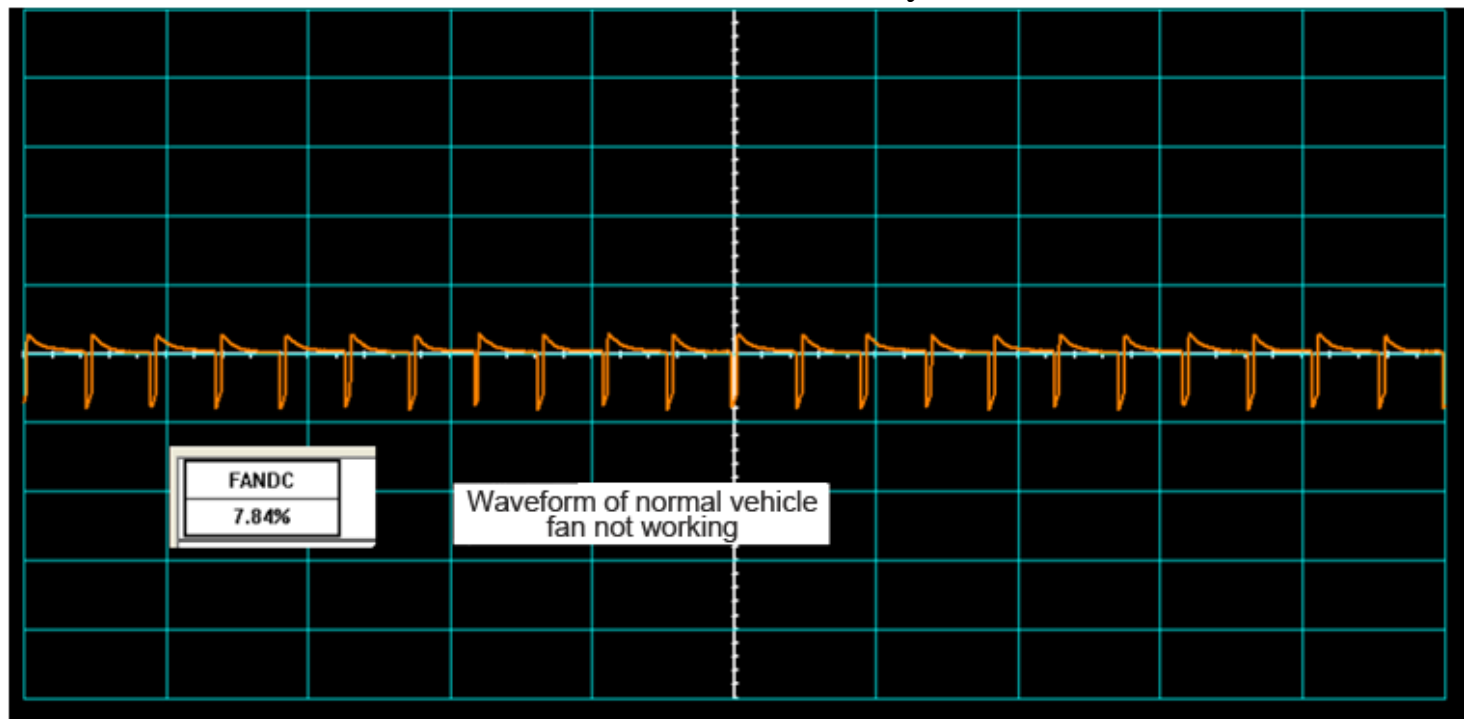

FIG. 3 Waveform of normal vehicle fan not working

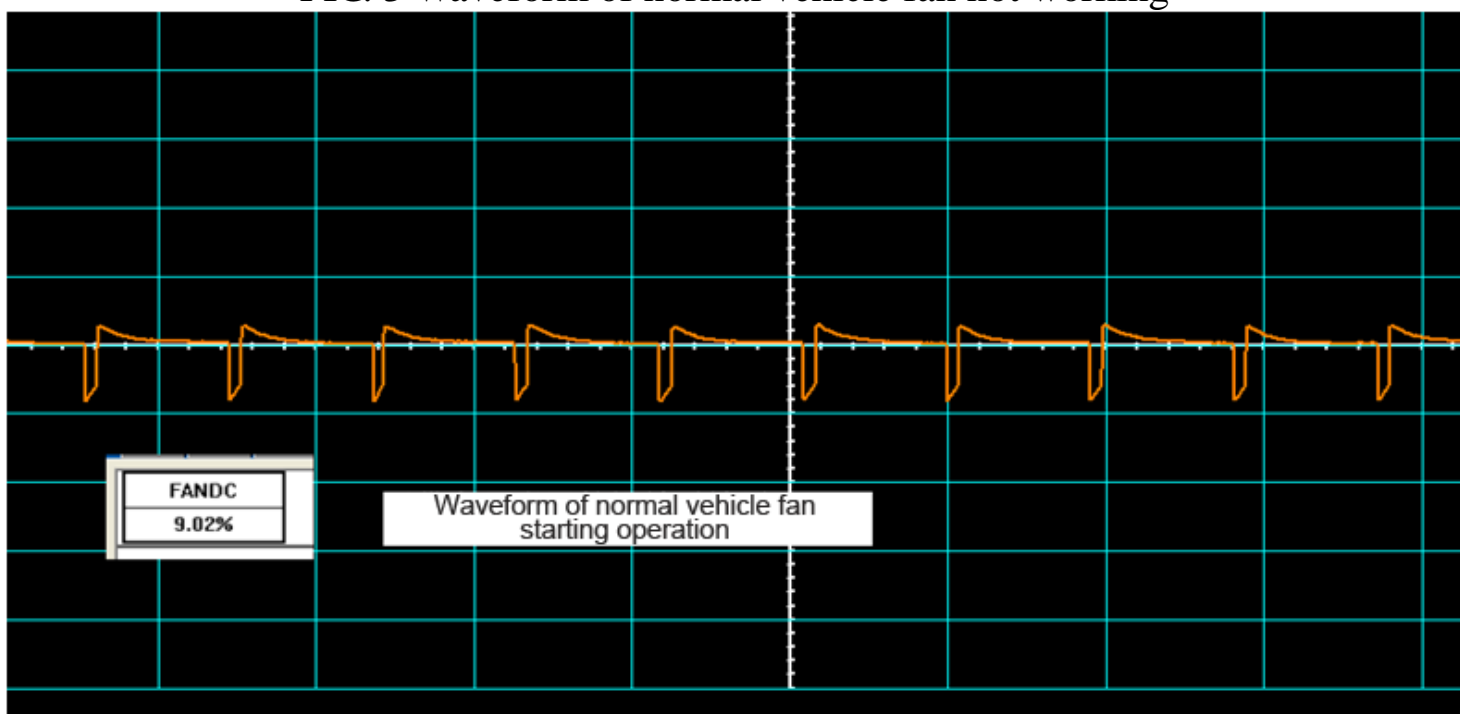

FIG. 4 Waveform of normal vehicle fan starting operation 


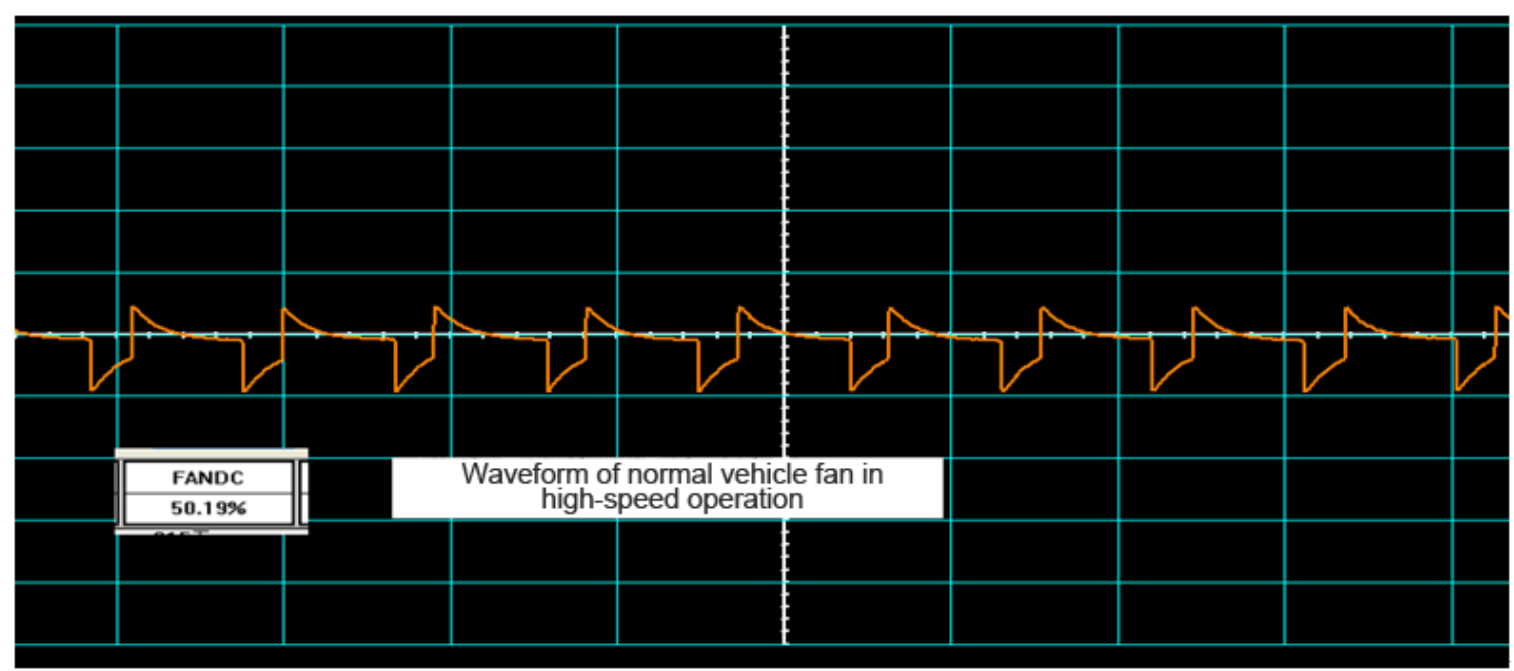

FIG. 5 Waveform of normal vehicle fan in high-speed operation

\section{Conclusion}

By analyzing the reasons for the high water temperature of the engine, the possible faulty parts were found. Through the real vehicle testing, the reasons of the failure were decomposed gradually, and it was found that the BCM failure was caused by the program upgrading, and the failure was completely eliminated finally. Through the test, the problem of high water temperature of the engine was found. According to the reasons mentioned in the paper, the maintenance method in the test was used to solve and troubleshoot one by one. The fault diagnosis method has certain reference significance to the problem of high water temperature of the engine, and whether BCM is normal in case of similar failures can be considered.

\section{References}

[1] Zhang Mengyun, Pan Jianfeng, Xue Hong, Li Detao. Research on fault diagnosis of diesel fuel oil supply system [J]. Agricultural Mechanization Research, 2011, 1 (8): 211-214.

[2] Zheng Taixiong, Zhang Yu, Li Yongfu. Research review of diagnosis methods for engine misfire failure in automobile engines [J]. Journal of Automation, 2014, 4 (43): 509-527.

[3] Yin Lili, Chen Deyun, Gu Hengwen, Wang Weiying. Anomaly detection method for gas turbine based on sensor network [J]. Journal of Aviation Dynamics, 2011, 1 (33): 39-47.

[4] Li Yunlong, Wang Xiangqing, Wang Jing, et al. Simulation study on underhood cooling duct flow field [J]. Vehicle \& Power Technology, 2005(3):27-31.

[5] Yuan Xianyi, Gu Zhengqi, Yang Yi. Numerical simulation analysis of engine room heat dissipation [J]. Automobile Engineering, 2009(9): 843-847

[6] Zhou Jianjun, Yang Kun. Application of numerical simulation in vehicle thermal management [J]. Shanghai Automobile, 2009(7):16-17.

[7] Liang Xiaobo .Automotive thermal management analysis using one-dimensional /threedimensional coupling simulation [J]. Automotive Engineering, 2010(9):793-798.

[8] Zhang Baoliang, Fan Qinyin. One-dimensional and three-dimensional coupling simulation of vehicle thermal management [J]. Automotive Engineering, 2011, 33(6): 492-496.

[9] Deng Yibin, Huang Ronghua, wWng Zhaowen. Vehicle thermal management system and its research [J]. Automotive Engineer, 2011(1):52-62.

[10] Guo Xinmin, Xing Juan, Yuan Yanli. Research on the electric cooling fan control system of 
automobile engine [J]. Automotive Electrical, 1996(5):13-15.

[11] Zhai Li, Qi Zicheng, Wang Xinyuan. Microcontroller control of automotive engine cooling system [J]. Agricultural Mechanization Research, 2001(2):38-39.

[12] Lu Guangfeng, Guo Xinmin, Sun Yunzhu. Development and status of automotive engine cooling system [J]. Agricultural Mechanization Research, 2002(2): 129-131.

[13] Fu Shouyu, Guo Xinmin, Zhang Kun. Design and test of double loop cooling system of diesel engine [J]. Journal of internal combustion engine, 2010(3): 265-268. 